\title{
CARBONO RETENIDO POR LA HOJARASCA Y RAÍCES EN DIFERENTES USOS DEL SUELO EN LA REGIÓN DEL CHACO SEMIÁRIDO
}

\section{CARBON RETAINED BY LITTER AND ROOTS IN DIFFERENT LAND USES IN THE SEMIARID REGION OF CHACO PROVINCE}

Flora E. Céspedes Flores ${ }^{1,2 *}$, Juan A. Fernández ${ }^{1}$, Laura Giménez ${ }^{1}$, Edgardo A. Leonhardt ${ }^{3}$, Aldo C. Bernardis $^{1}$

${ }^{1 *}$ Facultad de Ciencias Agrarias, Universidad Nacional del Nordeste (UNNE), Sargento Cabral 2131. C.P. 3400, Pcia. Corrientes, República Argentina.

2 Estación Experimental Agropecuaria INTA, Colonia Benítez, Av. Marcos Briolini S/N. C.P. 3505, Pcia. Chaco, República Argentina.

3 Agencia de Extensión Rural Pampa del Infierno, INTA Sáenz Peña, 9 de Julio N 558, Pampa del Infierno, C.P. 3708, Pcia. Chaco, República Argentina.

* Autor para correspondencia E-mail: fcespedes@agr.unne.edu.ar; cespedes.flora@inta.gob.ar

\section{RESUMEN}

Las prácticas de conservación agrícola y diversos sistemas de producción tradicionales pueden incrementar considerablemente el carbono orgánico en suelos. Con el objetivo de evaluar la acumulación de carbono en biomasa de raíces y hojarasca con diferentes usos del suelo, se realizó un ensayo en el oeste de la provincia del Chaco, Argentina. Los usos de la tierra (tratamientos) evaluados fueron: pastizal natural, pastura implantada, sistema silvopastoril, bosque nativo semiárido y campo agrícola. Se realizaron calicatas a lo largo de una transecta y se muestrearon los estratos: $0-0,05 ; 0,05-$ 0,$15 ; 0,15-0,3 ; 0,3-0,6 \mathrm{~m}$. Se utilizó un diseño en bloques completos al azar con 3 repeticiones. Los resultados fueron analizados con ANOVA y Test de Tukey $(\mathrm{p} \leq 0,05)$. El carbono en hojarasca fue mayor en la pastura $\left(670,7 \mathrm{~kg} \mathrm{ha}^{-1}\right)$ y en el sistema silvopastoril $\left(944,1 \mathrm{~kg} \mathrm{ha}^{-1}\right)$ comparado con el pastizal, el bosque nativo y el campo agrícola, que registraron valores de $300,3 \mathrm{~kg} \mathrm{ha}^{-1} ; 200,3 \mathrm{~kg} \mathrm{ha}^{-1}$; y 500,24 kg ha-1, respectivamente. El aporte de carbono de las raíces fue mayor en los dos primeros estratos muestreados $(0-0,05 \mathrm{~m}$ y $0,05-0,15 \mathrm{~m})$. El uso de bosque nativo registró valores de 3156,9 y $2779,2 \mathrm{~kg} \mathrm{ha}^{-1}$, mientras que el sistema silvopastoril alcanzó 2448,4 y $1228,9 \mathrm{~kg} \mathrm{ha}^{-1}$ a los 0,05 m y 0,15 $\mathrm{m}$ de profundidad, respectivamente. Estos dos usos son los que registraron el mayor aporte de carbono por raíces.

Palabras clave: pastizal, sistema silvopastoril, bosque nativo, stock de carbono, Chaco semiárido.

\section{ABSTRACT}

Agricultural conservation practices and various traditional production systems can significantly increase organic carbon in soils. A study was carried out in order to evaluate the accumulation of carbon in root and litter biomass with different land uses. The essay was conducted in the west of the Chaco province, Argentina. The following land uses (treatments) were evaluated: natural grassland, pasture, silvopastoral system, semi-arid native forest and cultivated field. Pits were made along a transect and the following strata were sampled: 0 to $0.05 ; 0.05$ to $0.15 ; 0.15$ to 0.3 ; and 0.3 to $0.6 \mathrm{~m}$. A randomized complete block design was used, with 3 replicates. The results were analyzed

Recibido: 27 abril 2018. Aceptado: 14 de junio de 2018. 
with ANOVA and Tukey test ( $\mathrm{p} \leq 0.05)$. Litter carbon was higher in the pasture $\left(670.7 \mathrm{~kg} \mathrm{ha}^{-1}\right)$ and silvopastoral system $\left(944.1 \mathrm{~kg} \mathrm{ha}^{-1}\right)$ compared to values recorded in the natural grassland, native forest and cultivated field, which reached $300.3 \mathrm{~kg} \mathrm{ha}^{-1} ; 200.3 \mathrm{~kg} \mathrm{ha}^{-1}$; and $500.24 \mathrm{~kg} \mathrm{ha}^{-1}$, respectively. The carbon contribution from the roots was greater in the first two strata sampled $(0$ to $0.05 \mathrm{~m}$ and 0.05 to $0.15 \mathrm{~m}$ ). The native forest recorded values of 3156.9 and $2779.2 \mathrm{~kg} \mathrm{ha}^{-1}$, while the silvopastoral system reached values of 2448.4 and $1228.9 \mathrm{~kg} \mathrm{ha}^{-1}$ at $0.05 \mathrm{~m}$ and $0.15 \mathrm{~m}$ depth, respectively. These two land uses recorded the highest root contributions of carbon.

Key words: grassland, silvopastoral system, native forest, carbono stock, arid Chaco

\section{INTRODUCCIÓN}

En los últimos dos siglos, el hombre modificó los ecosistemas a tasas desconocidas, afectando la biodiversidad, los flujos de energía, la producción y distribución de bienes y servicios, en algunos de manera positiva y en otros negativamente (Augustine et al., 2018). Todas estas alteraciones forman parte del fenómeno denominado Cambio Climático Global y representan un desafío para la comunidad científica, debido a la necesidad de brindar soluciones prácticas a estos problemas. Las actividades industriales y agropecuarias afectaron la concentración de dióxido de carbono $\left(\mathrm{CO}_{2}\right)$ en la atmósfera, a través de la quema de combustibles fósiles y la deforestación (Martino, 2007; Augustine et al., 2018).

Diversos estudios científicos proponen y analizan metodologías para reducir las emisiones de $\mathrm{CO}_{2}$ a la atmósfera y/o captar o secuestrar carbono en los ecosistemas (Lal, 2007). Fijar carbono en los ecosistemas terrestres no solo contribuye a disminuir la concentración de $\mathrm{CO}_{2}$ atmosférico, sino que incrementa las reservas de carbono y otros nutrientes del suelo, mejorando la fertilidad y la capacidad de producción de bienes y servicios (IPCC, 2003).

En la Provincia del Chaco (Argentina), la mayoría del territorio es de propiedad privada, las estrategias de manejo para aumentar el secuestro de carbono y la provisión de servicios ecosistémicos deben necesariamente ir acompañadas de planteamientos productivos atractivos (rentables) para los productores agropecuarios. Es importante conocer el historial de uso de la tierra, debido a que el uso inicial condiciona significativamente los cambios en las existencias de carbono en el suelo. No se producirá la misma fijación de carbono en un lugar si el uso inicial fue un cultivo, un pastizal o un bosque (Rodriguez et al., 2015; Etchevers et al., 2016).

El almacenamiento de carbono en los suelos es el balance entre la incorporación de material vegetal muerto (hojarasca y raíces) y las pérdidas durante los procesos de descomposición y mineralización (respiración heterotrófica) (Reyes et al., 2008).
El objetivo de esta investigación es evaluar el aporte de carbono de la biomasa de hojarasca y raíces en los usos de suelo más comunes en el oeste chaqueño de la Argentina..

\section{MATERIALES Y MÉTODOS}

El estudio se llevó a cabo en un área triangular de aproximadamente 250.000 hectáreas ubicadas en la porción oeste de la provincia del Chaco, Argentina, en la zona comprendida por las

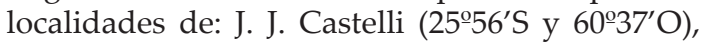

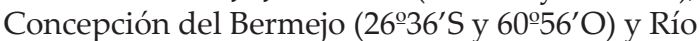
Muerto ( $26^{\circ} 18^{\prime} \mathrm{S}$ y $\left.61^{\circ} 39^{\prime} \mathrm{O}\right)$. Los establecimientos rurales de la zona se caracterizan por tener un $50 \%$ de cobertura forestal, con diferentes usos del suelo (agrícolas y ganaderos). La ganadería bovina (animales cruzas de razas Brahman, Braford y Brangus) se realiza sobre pastizales naturales manejando el rodeo (rebaño) con periodo de descanso del pastizal.

Esta región del Chaco tiene un relieve llano con suave pendiente hacia el sureste. El clima es subtropical continental, cálido, con estación seca marcada en invierno. La temperatura media anual es de $21,5^{\circ} \mathrm{C}\left(46^{\circ} \mathrm{C}\right.$ en verano y $6^{\circ} \mathrm{C}$ en invierno), las precipitaciones medias anuales varían entre $700-800 \mathrm{~mm}$ con un régimen estival. El material originario de suelo es de tipo sedimentario, resultante de un transporte mixto eólico-hídrico denominados limoloésicos (Morello et al., 2012). Las especies más representativas del bosque nativo son: Schinopsis lorentzii (quebracho colorado santiagueño), Aspidosperma quebracho blanco (quebracho blanco), Ziziphus mistol (mistol), Prosopis ruscifolia (itín), Schinus molle (molle), Celtis tala (tala). Se utiliza en el sistema de manejo silvopastoril de un bosque nativo, una densidad aproximada de 140 árboles $\mathrm{ha}^{-1}$, que tienen un arreglo natural, dado por la competencia inter específica sin intervención del hombre, considerando que los bosques de la zona evaluada provienen del raleo de especies por el aprovechamiento de la madera en el bosque nativo.

La sucesión de los ambientes más comunes en el oeste del Chaco, se inició particularmente del bosque nativo o de pastizal natural, y estos 
fueron cambiando de las siguientes maneras: a) a partir del bosque nativo que pasó a un manejo silvopastoril con pastura implantada de Panicum maximum cv. Gatton panic; b) bosque nativo que pasó a uso agrícola, principalmente cultivos de soja en siembra directa con diferentes rotaciones de gramíneas; c) ambientes de pastizal natural que cambiaron mediante la introducción de gramíneas subtropicales como $P$. maximum $\mathrm{cv}$. Gatton panic implantadas mediante el pasaje del rolo triturador y posterior quema (Silberman et al., 2015). Utilizando la variabilidad de los ambientes mencionados anteriormente, en el presente estudio se inició una evaluación de los mismos para observar lo que ocurre con la acumulación de carbono en hojarasca y raíces, en el período de junio 2011 a junio 2014.

Los tratamientos propuestos fueron los diferentes usos del suelo: Bosque nativo (BN), Pastizal natural de Elyonurus muticus (PN), Sistema Silvopastoril con las especies arbóreas más representativas del bosque, con pastura de $P$. maximum cv. Gatton panic (SSP), Campo agrícola: cultivo de soja con siembra directa (CA), y Pastura implantada de P. maximum cv. Gatton panic (PI)

En todos los usos del suelo estudiados se realizaron transectas diagonales equidistantes, donde se cavaron 3 calicatas de muestreo hasta los $0,6 \mathrm{~m}$ y se tomaron las muestras de suelo. La profundidad de muestreo fue definida en base a resultados obtenidos por Céspedes Flores et al. (2012) y además según las referencias bibliográficas a partir de esa profundidad del perfil de suelo, el carbono almacenado es menos susceptible a cambios y su modificación es a largo plazo, tal como lo fundamentan diversos autores (Piñeiro et al., 2006; IPCC, 2014; Rodriguez et al., 2015).

En cada calicata se muestrearon los estratos: 0-0,05 m; 0,05-0,15 m;0,15-0,3 m;0,3-0,6 m. En cada uso del suelo se estudió el contenido de carbono en raíces y hojarasca. La hojarasca (hojas senescentes depositadas sobre el suelo) fue muestreada por medio de la recolección en cada uso del suelo usando cuadros estandarizados de $1 \mathrm{~m}^{2}$, luego se determinó la MS a $65^{\circ} \mathrm{C}$.

El muestreo en el uso de CA fue antes de la cosecha, momento en el cual la biomasa vegetal es mayor y ya se produjo el mayor crecimiento de raíces del cultivo establecido, y en los demás usos del suelo fue en junio cuando las temperaturas son bajas y finalizó el período de crecimiento. Las raíces fueron separadas de la muestra de suelo por lavados, utilizando un tamiz con malla de $2 \mathrm{~mm}$ (Acosta et al., 2002), y las raíces menores se excluyeron por no diferenciarse del material orgánico en descomposición (IPCC, 2003; Trumper et al., 2009). Luego se llevaron a estufa a $65^{\circ} \mathrm{C}$ hasta peso constante para determinar materia seca (MS).

El cálculo de contenido de carbono en la biomasa de hojarasca y raíces se realizó considerando que es el $45 \%$ de la MS, entonces según Cruz-Flores y Etchevers (2011), y el IPCC (2003):

$$
\mathrm{C}=\mathrm{B} * 0,45 \text {; }
$$

dónde $\mathrm{C}=$ Contenido de carbono $\left(\mathrm{kg} \mathrm{ha}^{-1}\right) ; \mathrm{B}$ $=$ Biomasa radical $\left(\mathrm{kg} \mathrm{MS} \mathrm{ha}^{-1}\right) ; 0,45=$ constante, proporción de carbono en la biomasa vegetal, asumido por convención del IPCC.

Se utilizó un diseño en bloques completos al azar con 4 bloques representados por cada uno de los campos donde se encontraban todos los tratamientos (usos del suelo) y 3 repeticiones en cada uno. Con los datos se realizó un análisis de varianza y comparación de medias con un test de Tukey al 5\%, utilizando el software estadístico InfoStat (Di Rienzo et al., 2012).

\section{RESULTADOS Y DISCUSIÓN}

Los resultados obtenidos del contenido de carbono en hojarasca, muestran que los tratamientos PI y SSP fueron los de mayor retención de carbono, con valores de 670,7 y $944,1 \mathrm{~kg} \mathrm{ha}^{-1}$ respectivamente, diferenciándose significativamente $(p \leq 0,05)$ con el resto de los tratamientos (Fig. 1). Entre los tratamientos de $B N, P N$ y $C A$ no se registraron diferencias significativas $(p \geq 0,05)$ en la retención de carbono por la hojarasca. Cabe destacar que los valores tan bajos obtenidos en el tratamiento de $\mathrm{BN}$ se deben a que es un bosque nativo xerófilo, es decir son especies representativas de zona semiárida, que no generan demasiada biomasa, que al ser una zona de altas temperaturas la tasa de descomposición del material orgánico es mayor.

El tratamiento SSP no se diferenció significativamente del PI, debido a que el mayor aporte de la biomasa de hojarasca en el SSP lo realizó la pastura de $P$. maximum, y en menor grado los árboles xerófilos que crecen en el sitio; se debe tener en cuenta que el animal no sólo pastorea la pastura sino que ramonea los árboles, lo que propicia una reducción en la producción de hojarasca.

Los valores de hojarasca obtenidos fueron similares a los observados en trabajos por Céspedes Flores et al. (2010) quiénes estudiaron en el parque chaqueño húmedo un pastizal de Sorghastrum setosum y una pastura de Cynodon nlemfuensis, en concordancia con los valores de Cruz-Flores y Etchevers (2011) y Schöning et al. (2006). D'acunto et al. (2012) obtuvieron en la zona de La Pampa (Argentina) valores similares de carbono en usos agrícolas con cultivos de 


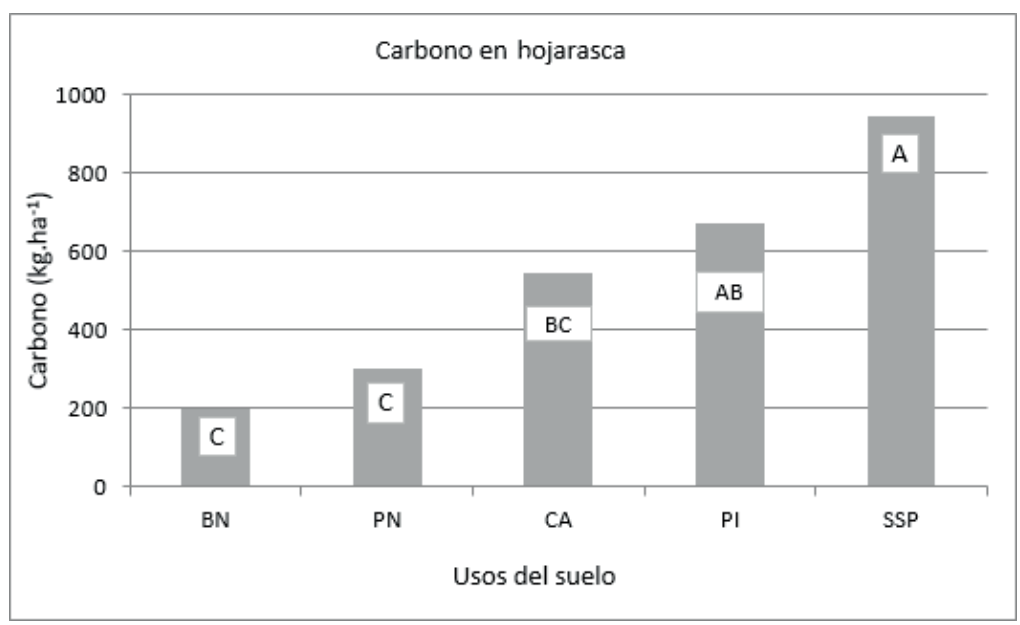

Fig. 1. Carbono retenido por la hojarasca en Bosque Nativo (BN), Campo Agrícola (CA), Pastizal Natural (PN), Pastura Implantada (PI) y Sistema Silvopastoril (SSP) en el oeste del Chaco. Letras diferentes indican diferencias significativas $(p \leq 0,05)$.

Fig. 1: Carbon retained by litter in Native Forest (BN), Cultivated Field (CA), Natural Grassland (PN), Pasture (PI) and Silvopastoral System (SSP) in the western Chaco. Different letters indicate significant differences $(\mathrm{p} \leq 0.05)$.

sorgo, maíz y soja. El aporte de carbono en la hojarasca en el CA, con el sistema de labranza conservacionista se realizó un aporte importante al stock de carbono, lo cual puede deberse a que tratándose de una siembra bajo labranza cero el material senescente se acumula, ya que en un cultivo de soja lo que se recolecta es la semilla y no la planta entera, por lo cual campaña tras campaña y dependiendo de los grupos de maduración que se utilicen ese material senescente va acumulándose, no es como el caso de la pastura o de árboles que pueden ser ramoneados por ganado, y por lo tanto no se aporta al suelo. Los muestreos se realizaron en el momento de mayor aporte del cultivo, luego de la cosecha.

La distribución de la PI genera mayor cobertura y atenúa el efecto del clima sobre la hojarasca al ser una pastura monofítica de desarrollo más homogéneo, esto no se observó para el caso de $\mathrm{PN}$ en donde, debido a condiciones naturales del ambiente y del pastizal de E. muticus evaluado, habían amplios sectores con baja densidad de pastizal, ya que esta especie predominante forma matas, y el espacio intermata está cubierto por otras especies de menor volumen de biomasa tanto aérea como de hojarasca, pero de mayor calidad forrajera que el pastizal mencionado (Sánchez Cárdenas et al., 2008).

En cuanto a los resultados del contenido de carbono de la biomasa radicular subterránea (Fig. 2) en BN el estrato de 0,05 m $(3156,9 \mathrm{~kg}$ $\left.h^{-1}\right)$ se diferenció significativamente $(p \leq 0,05)$ de los estratos de 0,30 y $0,60 \mathrm{~m}(2495$ y 2526 $\left.\mathrm{kg} \mathrm{ha}^{-1}\right)$. En el SSP el estrato de 0,05 m superó estadísticamente $\left(2448,4 \mathrm{~kg} \mathrm{ha}^{-1}\right)$ al resto de los estratos muestreados.

El CA fue el tratamiento que menos carbono retuvo en raíces. Presentó en comparación con los demás usos, para los estratos $0,05 \mathrm{~m}$ y 0,15 m (558 y $654 \mathrm{~kg} \mathrm{ha}^{-1}$, respectivamente) que se diferenciaron significativamente $(p \leq 0,05)$ de los 0,60 m (379 $\left.\mathrm{kg} \mathrm{ha}^{-1}\right)$ de profundidad (Fig. 2).

Debería considerarse que el CA se trata de cultivo de soja, familia de las Fabaceae, tiene raíz pivotante a diferencia de las gramíneas o poáceas que presentan raíz fibrosa, por lo cual el aporte de carbono de las raíces es diferente, a su vez se debe tener en cuenta que se trata del oeste chaqueño, que es una región semiárida con suelos con bajo contenido de materia orgánica $(1,08-1,06 \%)$ y que por las altas temperaturas y condiciones climáticas presenta una alta descomposición (Silberman et. al., 2015) (Fig. 2).

En el tratamiento de $\mathrm{PN}$ se presentaron diferencias estadísticas $(p \leq 0,05)$ de los $0,05 \mathrm{~m}$ y $0,15 \mathrm{~m}$ de profundidad respecto al resto de los estratos. En PI el estrato de 0,05 m (1834 $\left.\mathrm{kg} \mathrm{ha}^{-1}\right)$ fue significativamente superior al de los $0,30 \mathrm{~m}$ (1316 kg ha-1) de profundidad (Fig. 2).

En todos los usos del suelo en los primeros dos estratos de suelo evaluados, se retiene aproximadamente el $80 \%$ de carbono, en concordancia con lo obtenido por Rodriguez et al. (2015), quienes hallaron los mayores valores de carbono en diferentes usos del suelo en los primeros 

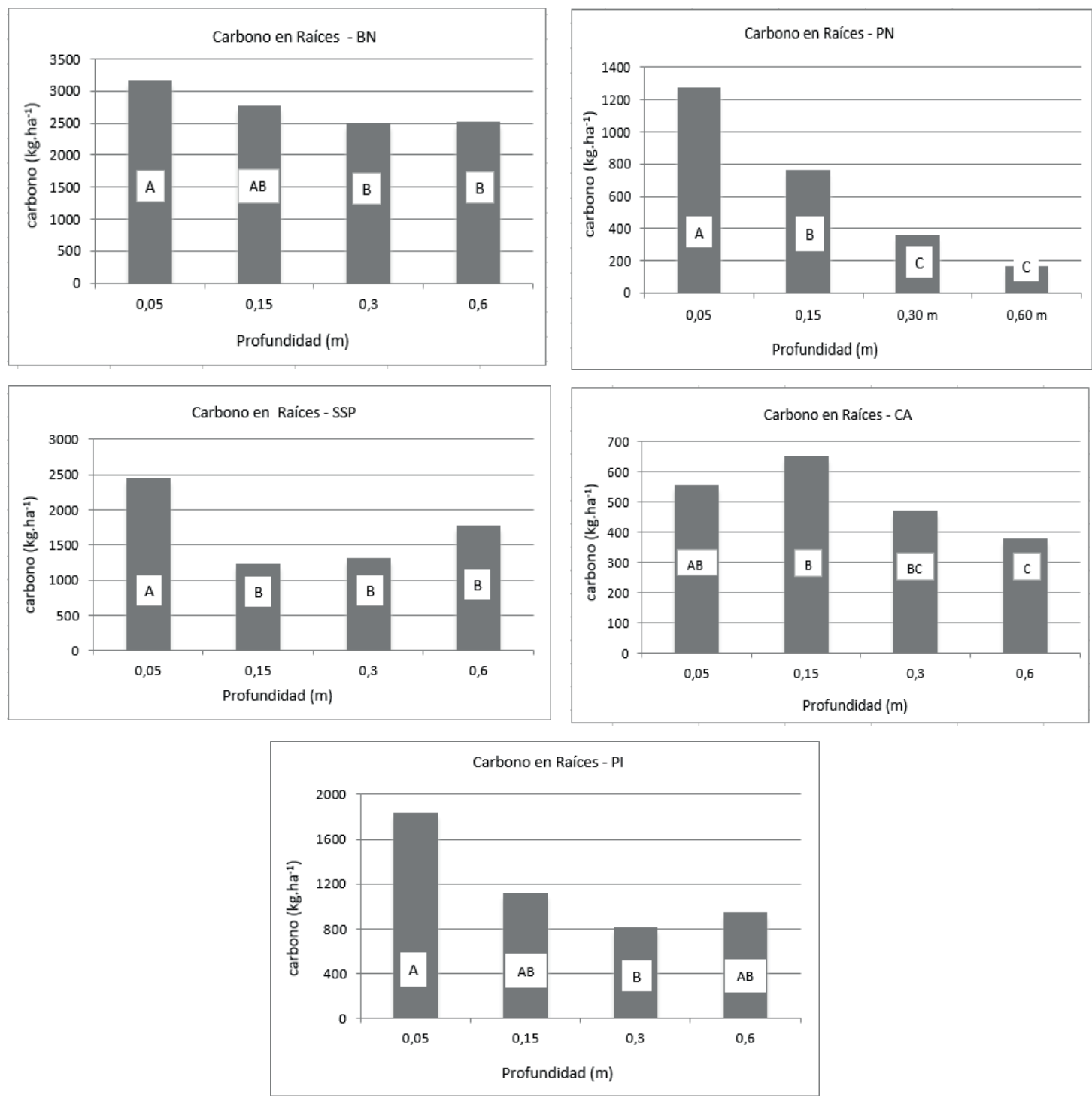

Fig. 2. Carbono en raíces de Bosque Nativo (BN), Campo Agrícola (CA), Pastizal Natural (PN), Pastura Implantada (PI) y Sistema Silvopastoril (SSP). Letras diferentes indican diferencias significativas entre profundidades $(p \leq 0,05)$.

Fig. 2. Carbon in roots of Native Forest (BN), Cultivated Field (CA), Natural Grassland (PN), Pasture (PI) and Silvopastoral System (SSP). Different letters indicate significant differences between depths $(\mathrm{p} \leq 0.05)$.

$20 \mathrm{~cm}$ de profundidad en Balcarce, provincia de Buenos Aires, en Argentina. Los resultados obtenidos en el aporte de carbono de la biomasa radicular subterránea (Fig. 2), coincidieron con lo observado por Ansín et al. (1999), Pucheta et al. (2004) y Cruz-Flores y Etchevers (2011) con diferentes usos del suelo tanto ganaderos como agrícolas, quiénes encontraron una variación en la distribución superficial de las raíces en el perfil de suelo, destacando que el mayor aporte de carbono hecho por las raíces fue en los primeros $0,15 \mathrm{~m}$. Las especies con sistema radicular profundo como las gramíneas constituyen una opción para incrementar en buena medida la acumulación de carbono, ya que redistribuyen el carbono en el perfil hasta el metro de profundidad, según Cespedes Flores et al. (2012).

En la Fig. 3, se puede observar el aporte total que realiza cada uso del suelo, el aporte de carbono de las raíces y de la hojarasca, el BN es el de mayor aporte significativo $\left(11157,52 \mathrm{~kg} \mathrm{ha}^{-1}\right)$ total, porque tuvieron raíces gruesas de gran aporte en cuanto a peso de MS total de las mismas. El que siguió en cuanto a aporte total fue el SSP (7711,04 kg.ha-1), 


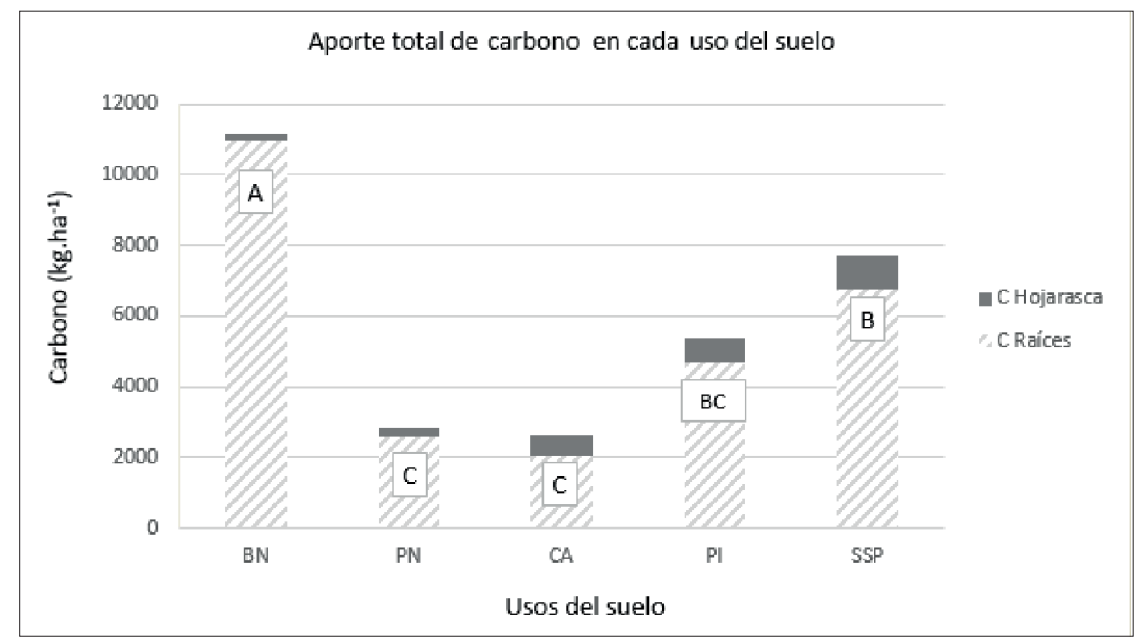

Fig. 3. Aporte total de carbono en cada uso del suelo evaluado (BN- PN- CA- PI- SSP). Letras diferentes indican diferencias significativas entre profundidades $(p \leq 0,05)$.

Fig. 3. Total contribution of carbon in each land use evaluated (BN-PN- CA- PI- SSP). Different letters indicate significant differences between depths $(\mathrm{p} \leq 0.05)$.

que no se diferenció significativamente del PI, y los usos con aportes totales menores fueron el PN y CA que no se diferenciaron estadísticamente (2861,77 y 2606,93 $\mathrm{kg} \mathrm{ha}^{-1}$, respectivamente).

Los árboles del BN son una fuente de aporte de gran importancia como contribuyente al C fijado en el sistema, se podría considerar medir únicamente árboles pero el costo de un muestreo destructivo de los mismos es elevado (CuellarBautista y Zanabria Mallqui, 2015).

Es esperable que el incremento de $\mathrm{CO}_{2}$ atmosférico y la temperatura afecten la productividad, la composición de especies, biogeoquímica, y además la cantidad y calidad de forraje disponible para los herbívoros en los ecosistemas de pastizales naturales (Augustine et al., 2018).

Augustine et al. (2018), encontraron en Norteamérica, que tanto el $\mathrm{CO}_{2}\left(\mathrm{CO}_{2}\right.$ equivalente$\mathrm{eCO}_{2}$ ) como el calentamiento global afectan la química del tejido vegetal a través de múltiples vías directas e indirectas. Para las seis especies forrajeras más abundantes de un pastizal natural en Norteamérica, en un ensayo de 7 años, el calentamiento global y el $\mathrm{eCO}_{2}$ aumentaron la producción de forraje en un $38 \%$ y redujeron el contenido de $\mathrm{N}$ en un $13 \%$. Para Pascopyrum smithii la digestibilidad in vitro de la MS (IVDMD) se redujo de 63,3 a 61,1\% IVDMD y el $\mathrm{N}$ de 1,25 a $1,04 \%$; tales cambios podrían tener consecuencias sustanciales en la velocidad a la que los rumiantes ganan peso durante la temporada de crecimiento primario en el ecosistema de pastizales más grande en Norteamérica.

\section{CONCLUSIONES}

El carbono retenido por la hojarasca en los tratamientos de pastura y sistema silvopastoril fue mayor que en el pastizal y bosque nativo bajo estudio.

El carbono retenido en raíces en los usos de suelo con bosque nativo y silvopastoril fueron los que acumularon más carbono en el perfil muestreado, y el menor fue en el uso agrícola.

Se encontró que el carbono retenido por las raíces fue mayor en los dos primeros estratos de suelo muestreados $(0-0,05 \mathrm{~m}$ en sumatoria con el estrato de 0,05-0,15 m), correspondiendo a un $80 \%$ aproximadamente del total de carbono de raíces en el perfil estudiado.

El aporte de carbono total de mayor aporte significativo se dio en el bosque nativo, los árboles son una fuente de aporte de gran importancia como contribuyente al C fijado en el sistema en la zona estudiada.

\section{RECONOCIMIENTOS}

Este trabajo se enmarcó en el Proyecto "Medición de la captura de carbono en sistemas pastoriles representativos del nordeste Argentino" de la Secretaría General de Ciencia y Técnica - Universidad Nacional del Nordeste (PI - 12A006), y en el Proyecto PSE -GEF 3623 (PNUD-ARG/10/G49-PNUMA 4B85) “Incentivo para la conservación de Servicios Ecosistémicos de importancia global". 


\section{LITERATURA CITADA}

Acosta, M., J.D. Etchevers, C. Monreal, K. Quednow, y C. Hidalgo. 2002. Un método para la medición del carbono en los compartimentos subterráneos (raíces y suelo) de sistemas forestales y agrícolas en terrenos de ladera en México. 15 p. En Simposio Internacional de Medición y Monitoreo de la Captura de Carbono en Ecosistemas Forestales. 18-20 de octubre. Valdivia, Chile. IUFRO. ISSN 1016-3263. World Series $\mathrm{N}^{\circ} 13$ : 14-18. Vienna, Austria.

Augustine, D.J., D.M. Blumenthal, T.L. Springer, D.R. Lecain, S.A. Gunter, and J. D. Derner. 2018. Elevated $\mathrm{CO}_{2}$ induces substantial and persistent decline in forage quality irrespective of warming in mixedgrass parierie. Ecological Society of America. Ecol. Appl. 28(3):721-735.

Ansín, O.E., E.M. Oyhamburu, E.A. Hoffmann, M.C. Vecchio, y M.C. Ferragine. 1999. Distribución de raíces en pastizales naturales y pasturas cultivadas de La Pampa Deprimida Bonaerense y su relación con la biomasa forrajera. Rev. Fac. Agron. La Plata. 103:141-148.

Céspedes Flores, F.E., J.A. Fernández, J.A. Gobbi, C. Roig, y A.C. Bernardis. 2010. Carbono retenido en la biomasa de hojarasca de pastizal de Sorghastrum setosum y pastura de Cynodon nlenfuensis. En $\mathrm{XXI}^{\circ}$ Reunión de comunicaciones científicas, técnicas y de extensión. Fac Cs Agrarias-UNNE. 25-27 de Junio. Corrientes, Argentina. (Resumen).

Céspedes Flores, F.E., J.A. Fernández, J.A. Gobbi, y A.C. Bernardis. 2012. Reservorio de carbono en suelo y raíces de un pastizal y una pradera bajo pastoreo. Rev. Fitotec. Mex. 35:79-86.

Cruz-Flores, G. y J.D. Etchevers-Barra. 2011. Contenidos de carbono orgánico de suelos someros en pinares y abetales de áreas protegidas de México. AGROCIENCIA 45 (8): 849-862.

Cuellar-Bautista, J. y R. Zanabria Mallqui. 2015. Carbono total almacenado en los depósitos de diferentes sistemas de uso de tierra del ecosistema alto andino, valle del Mantaro, Junín. Rev. Univ. Nac. Agraria La Molina. Lima, Perú. Ed. UNALM. ISSN 1997-6496. Xilema 28:43-52.
D'acunto, L. M. Semmartin, y C. Ghersa. 2012. Dinámica del carbono en cultivos de soja. ¿Influyen los vecinos? En Congreso de la Reunión Argentina de Ecología. Asociación Argentina de Ecología. 24-29 de Septiembre. Luján, Argentina. Disponible en http://www.conicet.gov.ar/new_scp/detalle. php? keywords $=\& i d=02662 \&$ inst $=$ yes\&congresos=yes\&detalles=yes\&congr_id $=1376142$ (Consulta 15 octubre 2015).

Di Rienzo, J.A., F. Casanoves, M.G. Balzarini, L. Gonzalez, M. Tablada, et al. 2012. Grupo InfoStat, FCA, Universidad Nacional de Córdoba, Argentina. 2012. Disponible en http://www.infostat.com.ar (Consulta 15 agosto 2014).

IPCC. 2014. Cambio climático 2014. R.K. Pachauri y L.A. Meyer (eds.) Informe de síntesis. Contribución de los Grupos de trabajo I, II y III al Quinto Informe de Evaluación del Grupo Intergubernamental de Expertos sobre el Cambio Climático. 157 p. IPCC, Ginebra, Suiza.

IPCC. 2003. Good practice guidance for land use, land-use change and forestry. $632 \mathrm{p}$. Institute for Global Environment Strategies, Kanagawa, Japan.

Lal, R., D.C. Reicosky, and J.D. Hanson. 2007. Evolution of the plow over 10,000 years and the rationale for no-till farming. Soil Tillage Res. 93:1-12.

Martino, D.L. 2007. El cambio climático y los costos de su mitigación. Material complementario del Informe mundial sobre desarrollo humano 2007-2008. Uruguay: El cambio climático aquí y ahora. 39 p. PNUD, Montevideo, Uruguay.

Morello, J., S. Matteucci, A. Rodríguez y M. Silva. 2012. Ecorregiones y complejos ecosistémicos Argentinos. 752 p. Facultad de Arquitectura Diseño Urbanismo. Universidad de Buenos Aires. Orientación Gráfica Editora, Buenos Aires, Argentina.

Piñeiro, G., J.M. Paruelo, and M. Oesterheld. 2006. Potential long-term impacts of livestock introduction on carbon and nitrogen cycling in grasslands of Southern South America. Global Change Biology 12:1267-1284.

Pucheta, E., I. Bonamici, M. Cabido, and S. Díaz. 2004. Below-ground biomass and productivity of a grazed site and a neighboring ungrazed exclosure in a grassland in central Argentina. Austral Ecol. 29:201-208. 
Reyes, G.C., H.H. Trejo, y J.L.M. Sánchez. 2008. Captura de carbono en un pastizal de La Ranchería Emiliano Zapata, Centro, Tabasco. Rev. de divulgación. División académica de Cs. Biológicas. ISSN 1665-0514. 36(14):12 Disponible en http://revistas.ujat.mx/index. php/kuxulkab/article/view/893/748 (Consulta 4 noviembre de 2016).

Rodriguez, S., C.C. Videla, E.C. Zamuner, L.I. Picone, N.N. Pose and N.O. Maceira. 2015. Cambios en propiedades químicas de un suelo molisol de la Región Pampeana Argentina con diferente historia de manejo. Chilean J. Agric. Anim. Sci., ex Agro-Ciencia 31(2): 137-148.

Sánchez Cárdenas, S., G. Crespo López, M. Hernández Chávez y Y. García. 2008. Acumulación y descomposición de la hojarasca en un pastizal de Panicum maximum y en un sistema silvopastoril asociado con Leucaena leucocephala. Zoot. Trop. 26(3):269273.
Schöning, I., K.U. Totsche, and I. Kögel-Knabner. 2006. Small scale spatial variability of organic carbon stocks in litter and solum of a forested Luvisol. Geoderma 136:631-642.

Silberman, J.E., A.L. Anriquez, J.A. Domínguez Núñez, C.G. Kunst y A.S. Albanesi. 2015. La cobertura arbórea en un sistema silvopastoril del Chaco y su contribución diferencial al suelo. Asociación Argentina de la Ciencia del Suelo. Cienc. Suelo 33(1):19-29.

Trumper, K., M. Bertzky, B. Dickson, G. Van der Heijden, M. Jenkins, y P. Manning. 2009. ¿La solución natural? El papel de los ecosistemas en la mitigación del cambio climático. 76 p. Informe del Programa de las Naciones Unidas para el Medio Ambiente. PNUMA, Cambridge, Reino Unido. 